\title{
INTEGRAL OVERRINGS \\ OF TWO-DIMENSIONAL GOING-DOWN DOMAINS
}

\author{
DAVID E. DOBBS AND MARCO FONTANA
}

(Communicated by Louis J. Ratliff, Jr.)

\begin{abstract}
It is proved that if $R$ is a 2-root closed two-dimensional going-down domain with no factor domain of characteristic 2 , then each integral overring of $R$ is a going-down domain. An example is given to show that the "2-root closed" hypothesis cannot be deleted.
\end{abstract}

\section{INTRODUCTION}

Let $R$ be a (commutative integral) domain. As in [6,12], we say that $R$ is a going-down domain if $R \subset T$ satisfies GD (in the sense of [17, p. 28]) for each domain $T$ containing $R$. Natural examples of going-down domains include Prüfer domains, domains of (Krull) dimension 1, and certain $D+M$ constructions (cf. [12, Corollary]). In some respect, the class $\mathscr{C}$ of going-down domains is well-behaved; for instance, being a going-down domain is a local property (cf. [6, Lemma 2.1]). Moreover, certain subclasses of $\mathscr{C}$ are known to be stable under integral extensions (cf. [15, Theorem 1.7; 10, Proposition 2.6]). However, $\mathscr{C}$ is not stable under integrality. Indeed, by analyzing a construction of Heinzer-Ohm [16, p. 6], Dobbs showed in [7, Example 2.1] that an integral extension of a two-dimensional valuation domain need not be a going-down domain. By applying the classical $D+M$ construction and [12, Corollary], we see for each $n, 3 \leq n \leq \infty$, that there exists an $n$-dimensional $R \in \mathscr{C}$ such that $R$ has an integral overring which is not in $\mathscr{C}$. (It follows from [8, Lemma 2.2(b)] that the domains $R$ in these examples are, in fact, divided domains, an important type of quasilocal going-down domain studied in [8].) Our main purpose in this note is to present two results which clarify the situation in the two-dimensional case.

In the positive direction, Theorem 2.2 shows that if $R$ is a 2-root closed two-dimensional going-down domain (and, hence, locally divided, in the sense of [8]), and if $R$ satisfies a mild condition (which holds if $\operatorname{char}(R)>2$ ), then each integral overring of $R$ is in $\mathscr{C}$. (The reader may wish to compare this

Received by the editors December 24, 1990.

1980 Mathematics Subject Classification (1985 Revision). Primary 13G05; Secondary 13A17, 13B02, 13B20, 13F05, $13 \mathrm{D} 99$.

Both authors were supported in part by NATO Collaborative Research Grant CRG 900113.

The second author was supported in part by Ministero dell'Università e della Ricerca Scientifica e Tecnologica (60\% Fund) and University of Tennessee Science Alliance. 
assertion with earlier results in the two-dimensional case: cf. [7, Corollary 3.5; 9, §3].) The work in $\S 2$ depends in part on adapting a proof of Angermüller [3, Main Lemma]; the reader may find it helpful to have a copy of [3] at hand while reading Lemma 2.1(b). In a negative vein, Example 3.1 constructs a two-dimensional divided (and, hence, going-down) domain $R$ (which is not 2 -root closed and may have arbitrary characteristic) having an integral overring which is not in $\mathscr{C}$. The work in $\S 3$ depends on pullback techniques, for which familiarity with [14] will be assumed.

\section{A POSITIVE RESUlT}

We begin by collecting some results of Angermüller. Lemma 2.1(a) simply restates [3, Lemma 1], while Lemma 2.1(b) follows from the proof of [3, Main Lemma]. (Our formulation of Lemma 2.1(b) deletes the hypotheses in [3, Main Lemma] that $A$ is one-dimensional Noetherian and that $B$ is the integral closure of $A$. To adapt the proof in [3], one also needs the well-known fact [5, Proposition 3, p. 329] that module-finite extensions of commutative rings satisfy the finite fiber property. For another extension of [3, Main Lemma], see [4, Lemma 2].) As usual, if $n>1$ is an integer, a domain $A$ is called $n$-root closed if $A$ contains each element $u$ of its quotient field such that $u^{n} \in A$.

Lemma 2.1 (Angermüller). Let $A$ be an $n$-root closed domain for some integer $n>1$, and let $B$ be an overring of $A$. Then:

(a) The conductor $(A: B)$ is a radical ideal of $B$.

(b) Assume, in addition, that $(A, M)$ is quasilocal, $B$ is module-finite over $R,(A: B)=M$, and that $B / N$ has a primitive nth root of unity, for some maximal ideal $N$ of $B$. Then $(B, M)$ is quasilocal.

It is convenient next to recall the following material from [8]. A domain $R$ is divided if $P R_{p}=P$ for each prime ideal $P$ of $R$; and $R$ is locally divided if $R_{M}$ is divided for each maximal (equivalently, each prime) ideal $M$ of $R$. Each locally divided domain is a going-down domain; the converse holds if $R$ is root closed (indeed, by the proofs in [8], if $R$ is seminormal), but is false in general. Also, a domain $R$ is treed if $\operatorname{Spec}(R)$, as a poset under inclusion, is a tree. Each going-down domain is treed [6, Theorem 2.2]; but, as is shown by an example of W. J. Lewis (cf. [13, Example 4.4]), the converse is false.

We may now state our main positive result.

Theorem 2.2. Let $R$ be a 2-root closed two-dimensional going-down domain with no factor domain of characteristic 2. Then each integral overring $S$ of $R$ is a going-down domain.

Proof. For each maximal ideal $M$ of $R, R_{M}$ is a 2-root closed going-down domain (of dimension at most 2, with no factor domain of characteristic 2). Moreover, it is easy to see that $S \in \mathscr{C}$ provided that each $S_{R \backslash M} \in \mathscr{C}$. Thus, we may suppose that $(R, M)$ is quasilocal.

By integrality, we can view $S$ as the direct limit of its family of module-finite $R$-subalgebras. Since [11, Corollary 2.7] establishes that direct limit preserves going-down domains, we may also suppose that $S$ is module-finite over $R$.

Consider the conductor $I=(R: S)$. By Lemma $2.1(\mathrm{a}), I$ is a radical ideal of $S$. Hence, $I$ is a radical ideal of $R$. However, by the above remarks, $R$ is 
quasilocal treed with, say, $\operatorname{Spec}(R)=\{0, P, M\}$, and so $I \in \operatorname{Spec}(R)$. As $S$ is a module-finite overring of $R, I \neq 0$; that is, $I$ is either $P$ or $M$.

Now, suppose that the assertion fails. Then there exists a domain $V$ containing $S$ such that $S \subset V$ does not satisfy GD. Since $R \subset V$ does satisfy $\mathrm{GD}$, an easy diagram chase shows that some height 1 prime of $R$ (namely, $P$ ) is not unibranched in $S$. (For this argument, one needs to observe that $R \subset S$ satisfies INC and that $\operatorname{dim}(S)=2$.)

We claim that $I \neq P$. Otherwise, consider $A=R_{P}$ and $B=S_{R \backslash P}$. Since $S$ is module-finite over $R$, we have $(A: B)=I_{R \backslash P}=P R_{P}$. However, since $R$ is a quasilocal 2-root closed (hence seminormal) going-down domain, it follows from the above remarks that $R$ is a divided domain. In particular, $P R_{P}=P$, and so $(A: B)=P$. By the hypothesis on $R$, no factor domain of $B$ can have characteristic 2 . Hence, we may apply Lemma 2.1 (b) with $n=2$, since $A$ is 2-root closed and $-1+N$ is a square root of unity in any factor domain $B / N$. This yields that $(B, P)$ is quasilocal, contradicting the fact that $P$ is not unibranched in $S$. This proves the claim. Hence, $I=M$.

We may again apply Lemma $2.1(\mathrm{~b})$, this time to $R \subset S$ and $n=2$, since no factor domain of $S$ can have characteristic 2. This yields that $(S, M)$ is quasilocal. It follows that each prime ideal of $S$ is a prime ideal of $R$. (In fact, $\operatorname{Spec}(S)=\operatorname{Spec}(R)$ as sets.) This contradicts the fact that $P$ is not unibranched in $S$, thus completing the proof.

The most immediate application of Theorem 2.2 is the following.

Corollary 2.3. Let $R$ be a 2-root closed two-dimensional going-down domain of characteristic $p>2$. Then each integral overring of $R$ is a going-down domain.

It will be shown in Example 3.1 that the "2-root closed" hypotheses in Theorem 2.2 and Corollary 2.3 (and the " $n$-root closed hypothesis" in Corollary 2.4) cannot be deleted.

The hypotheses of Theorem 2.2 were chosen so that the proof would not be overly technical. One may well ask for variants that relate to the formulation of Lemma 2.1(b) for arbitrary $n \geq 2$. In this regard, we close the section by offering the following result.

Corollary 2.4. Let $R$ be a two-dimensional going-down domain and $S$ an overring of $R$. Assume that at least one of the following two conditions holds:

(a) $S$ is module-finite over $R$, and for each nonzero prime ideal $N$ of $S$, there exists an integer $n>1$ such that $R$ is $n$-root closed and the quotient field of $S / N$ contains a primitive nth root of unity;

(b) $S$ is integral over $R$, and for each nonzero prime ideal $P$ of $R$, there exists an integer $n>1$ such that $R$ is $n$-root closed and the quotient field of $R / P$ contains a primitive nth root of unity.

Then $S$ is a going-down domain.

Proof. Given (a), the assertion follows as in the proof of Theorem 2.2. Given (b), one first reduces to the case of module-finite $S$ by using the fact that $\mathscr{C}$ is stable under direct limit; and the assertion then follows since, for module-finite $S$, (b) implies (a). 


\section{A COUNTEREXAMPLE}

The main purpose of this section is to produce an example of a two-dimensional going-down domain $R$ and a module-finite overring $S$ of $R$ such that $S$ is not a going-down domain. In view of Theorem 2.2, one expects that a construction of such $R$ should violate the "2-root closed" hypothesis. This is, in fact, how we proceed in Example 3.1, with a pullback construction that exploits the quadratic nature of the diagonal map $A \rightarrow A \times A$ for any commutative ring A.

In the following discussion, it will be convenient to let $(A, m, k)$ denote a quasilocal ring $A$ with maximum ideal $m$ and residue field $k=A / m$.

Example 3.1. There exists a two-dimensional going-down domain $R$ and a module-finite (hence integral) overring $S$ of $R$ such that $S$ is not a going-down domain. It can be arranged that $R$ have arbitrary (prime or zero) characteristic, indeed that $R$ contain any preassigned field. It can also be arranged that either $R$ be a divided domain or there exist $u \in S$ such that $S=R[u]$.

Proof. Consider two incomparable DVRs, $\left(V^{\prime}, m^{\prime}, K^{\prime}\right)$ and $\left(V^{\prime \prime}, m^{\prime \prime}, K^{\prime \prime}\right)$, of a field $F$. (For instance, if the desired $R$ is to contain a given field $k$, take commuting algebraically independent indeterminates $U$ and $V$ over $k$, and consider $F=k(U, V), V^{\prime}=k(U)[V]_{(V)}, V^{\prime \prime}=k(V)[U]_{(U)}, K^{\prime}=k(U)$, and $K^{\prime \prime}=k(V)$.) Put $D=V^{\prime} \cap V^{\prime \prime}$. By [17, Theorem 107], $D$ is a one-dimensional Bézout domain (in fact, a PID) with but two maximal ideals, $M^{\prime}=m^{\prime} \cap D$ and $M^{\prime \prime}=m^{\prime \prime} \cap D$; and $F$ is the quotient field of $D$. (Indeed, $D_{M^{\prime}}=V^{\prime}$ and $D_{M^{\prime \prime}}=V^{\prime \prime}$.) Next, let $\left(W^{\prime}, n^{\prime}, k^{\prime}\right)$ and $\left(W^{\prime \prime}, n^{\prime \prime}, k^{\prime \prime}\right)$ be two other DVRs having quotient fields $K^{\prime}$ and $K^{\prime \prime}$, respectively. (For instance, take $W^{\prime}=$ $k[U]_{(U)}$ and $W^{\prime \prime}=k[V]_{(V)}$.) Consider also a DVR, $(W, n, k)$, with quotient field $K$ canonically isomorphic to both $K^{\prime}$ and $K^{\prime \prime}$, so that $W$ is canonically isomorphic to both $W^{\prime}$ and $W^{\prime \prime}$ via isomorphisms that are compatible with the structures involving $k$ and $K$.

Let $T^{\prime}$ and $T^{\prime \prime}$ be the two-dimensional valuation domains of $F$ obtained via "Nagata composition" using the pullbacks

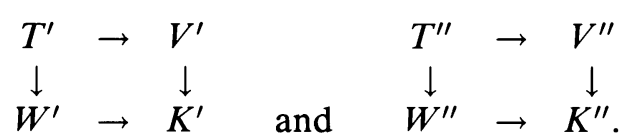

(In such pullback diagrams, we generally assume that the horizontal maps are the canonical inclusions and the vertical maps are the canonical epimorphisms.) Put $C=T^{\prime} \cap T^{\prime \prime}$. Notice that we have a pullback diagram

$$
\begin{array}{rll}
C & \rightarrow & D \\
\downarrow & & \downarrow \\
W^{\prime} \times W^{\prime \prime} & \rightarrow & D /\left(M^{\prime} \cap M^{\prime \prime}\right) \cong K^{\prime} \times K^{\prime \prime}
\end{array}
$$

where the isomorphism in the bottom row is given by the Chinese Remainder Theorem. Since $C$ and $D$ have the common ideal $M^{\prime} \cap M^{\prime \prime}$, they have the same quotient field, $F$.

Much more can be said about $C$, by using some results from [14]. Applying [14, Theorem 1.4] to the pullback diagram that defines $C$ gives a topological description of $\operatorname{Spec}(C)$ with the following order-theoretic impact. $C$ is twodimensional; besides the prime $0, C$ has but two maximal ideals (say, $N^{\prime}$ and 
$N^{\prime \prime}$ ) and but two height 1 prime ideals (say, $P^{\prime}$ and $P^{\prime \prime}$ ), where $P^{\prime} \subset N^{\prime}$, $P^{\prime \prime} \subset N^{\prime \prime}, P^{\prime}=M^{\prime} \cap C$, and $P^{\prime \prime}=M^{\prime \prime} \cap C$.

Consider the effect of applying [14, Proposition 1.9], by localizing the pullback diagram that defines $C$ at the multiplicative sets induced by (the complement of) the prime $P^{\prime}$. The result is a pullback diagram

$$
\begin{array}{ccc}
C_{P^{\prime}} & \rightarrow & D_{M^{\prime}}=V^{\prime} \\
\downarrow & & \downarrow \\
K^{\prime}=W_{W^{\prime} \backslash\{0\}}^{\prime} & \rightarrow & K^{\prime}
\end{array}
$$

It follows that $C_{P^{\prime}}=V^{\prime}$; similarly, $C_{P^{\prime \prime}}=V^{\prime \prime}$.

Next, let $\alpha$ be the composition of canonical surjections, $C \rightarrow W^{\prime} \times W^{\prime \prime} \rightarrow$ $W^{\prime}$. As $\operatorname{ker}(\alpha)=M^{\prime} \cap C=P^{\prime}$, a standard isomorphism theorem gives $C / P^{\prime} \cong$ $W^{\prime}$; similarly, $C / P^{\prime \prime} \cong W^{\prime \prime}$. Since $\alpha\left(N^{\prime}\right)=n^{\prime}$, an isomorphism theorem gives $N^{\prime} / P^{\prime} \cong n^{\prime} ;$ similarly, $N^{\prime \prime} / P^{\prime \prime} \cong n^{\prime \prime}$. This leads to canonical identifications $C / N^{\prime} \cong\left(C / P^{\prime}\right) /\left(N^{\prime} / P^{\prime}\right) \cong W^{\prime} / n^{\prime} \cong k^{\prime}$ and, similarly, $C / N^{\prime \prime} \cong k^{\prime \prime}$.

Next, we define $B$ by the pullback diagram

$$
\begin{aligned}
& B \rightarrow C \\
& \downarrow \\
& k \rightarrow \stackrel{\downarrow}{C} /\left(N^{\prime} \cap N^{\prime \prime}\right) \cong k^{\prime} \times k^{\prime \prime} .
\end{aligned}
$$

Notice that $B$ has the same quotient field as $C$, namely, $F$. Applying [14, Theorem 1.4] to the above pullback diagram, we see that $B$ is a two-dimensional quasilocal domain, with maximal ideal $N^{\prime} \cap N^{\prime \prime}$, and exactly two height 1 primes, $Q^{\prime}=P^{\prime} \cap B$ and $Q^{\prime \prime}=P^{\prime \prime} \cap B$. (In effect, the passage from $C$ to $B$ "glues" $N^{\prime}$ and $N^{\prime \prime}$ together.) Notice that $B$ is not treed, and so $B$ is not a going-down domain.

We can now define one of the rings whose existence is asserted in the statement of Example 3.1. Define $A$ by the pullback diagram

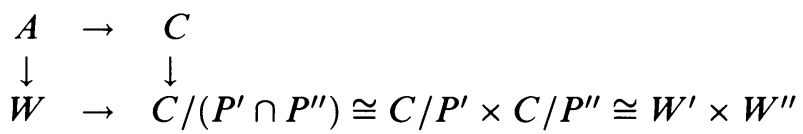

(The first isomorphism in the bottom row is available via the Chinese Remainder Theorem because $P^{\prime}$ and $P^{\prime \prime}$ are comaximal in $C$.) As above, the quotient field of $A$ is $F$. Also, one sees readily that $A \subset B$. (Indeed, if $a \in A$, there exists $c \in C$ such that $a-c \in\left(P^{\prime} \cap P^{\prime \prime}\right) \subset\left(N^{\prime} \cap N^{\prime \prime}\right)$, whence $\left.a \in B\right)$. Moreover, since the diagonal map allows us to view $W^{\prime} \times W^{\prime \prime}$ as an integral extension of $W$, it follows from [14, Corollary 1.5(5)] or a direct calculation that $C$ is integral over $A$; then, a fortiori so is $B$.

The above succession of pullbacks has finally produced a two-dimensional treed quasilocal domain, namely, $A$, with quotient field $F$. In fact, we see by applying [14, Theorem 1.4] to the definition of $A$ that the unique maximal ideal of $A$ is $N=N^{\prime} \cap A=N^{\prime \prime} \cap A$, and the unique height 1 prime of $A$ is $Q=P^{\prime} \cap A=P^{\prime \prime} \cap A=P^{\prime} \cap P^{\prime \prime}$. (Hence, since $A / Q$ is canonically isomorphic to $W$, we may identify their height 1 primes; that is, $N / Q=n$.) Moreover, since $A \subset B \subset C$, we have that $Q=Q^{\prime} \cap A=Q^{\prime \prime} \cap A$.

We next identify another important ring. Define $E$ by the pullback diagram

$$
\begin{aligned}
& E \rightarrow D \\
& \stackrel{\downarrow}{K} \rightarrow \stackrel{\downarrow}{D} /\left(M^{\prime} \cap M^{\prime \prime}\right) \cong D / M^{\prime} \times D / M^{\prime \prime} \cong K^{\prime} \times K^{\prime \prime} .
\end{aligned}
$$


Appealing to [14] as above, we see that $E$ has quotient field $F$; and that $E$ is quasilocal one-dimensional, with unique maximal ideal $e=M^{\prime} \cap E=M^{\prime \prime} \cap E=$ $M^{\prime} \cap M^{\prime \prime}$.

We claim that $E=A_{Q}$. To see this, first juxtapose the pullback diagrams that defined $A$ and $C$. The result is the pullback diagram

$$
\begin{aligned}
A & \rightarrow D \\
\downarrow & \stackrel{\downarrow}{\rightarrow} K \rightarrow \stackrel{D}{D}\left(M^{\prime} \cap M^{\prime \prime}\right) \cong K^{\prime} \times K^{\prime \prime}
\end{aligned}
$$

where the maps in the bottom row are the canonical inclusion and the diagonal. Since $E$ is the inverse image of $K$ under the surjection from $D$ to $K^{\prime} \times K^{\prime \prime}$, we now have the pullback diagram

$$
\begin{array}{rll}
A & \rightarrow & E \\
\downarrow & & \downarrow \\
W & \rightarrow & K=E / e
\end{array}
$$

Consider the effect of applying [14, Proposition 1.9], by localizing the above pullback diagram at the multiplicative sets induced by (the complement of) the prime $Q$. The result is a pullback diagram

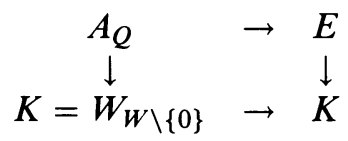

It follows that $A_{Q}=E$, as claimed.

Next, we claim that $A$ is a divided domain. It suffices to show that $Q A_{Q} \subset$ $A$. It will be convenient to let $J$ denote $Q A_{Q}$. Now, since $Q=P^{\prime} \cap A=$ $P^{\prime \prime} \cap A$, we have

$$
E=A_{Q} \subset C_{P^{\prime}} \cap C_{P^{\prime \prime}}=V^{\prime} \cap V^{\prime \prime}=D .
$$

In particular, $J \subset D$. Notice that the surjection $\pi: D \rightarrow K^{\prime} \times K^{\prime \prime}$ sends $Q$ to 0 because $Q \subset M^{\prime} \cap M^{\prime \prime}$. Hence, $\pi$ sends $J=Q E$ to 0 . The definition of $C$ yields that $J \subset C$; and that the canonical map from $C$ to $W^{\prime} \times W^{\prime \prime}$ sends $J$ to 0 . It follows from the definition of $A$ that $J \subset A$, thus proving the claim that $A$ is divided.

Before identifying the required $(R, S)$, we claim that $B=A\left[u_{1}, u_{2}\right]$, for suitable elements $u_{1}, u_{2}$. For this, it is enough to observe that $W^{\prime} \times W^{\prime \prime}$ is generated as a module over (the DVR) $W$ by $\{(1,0),(0,1)\}$, so that $B /\left(P^{\prime} \cap P^{\prime \prime}\right)$ can be generated as a $W$-module by two elements. In particular, $B$ is module-finite over $A$. If one wants that $A$ be divided, it suffices to take $(R, S)=(A, B)$. It remains to consider the case where one wants $S$ to be generated by one element as an $R$-algebra. For this, take $(R, S)$ to be either $\left(A\left[u_{1}\right], B\right)$ or $\left(A, A\left[u_{1}\right]\right)$, according as to whether $A\left[u_{1}\right]$ is or is not a going-down domain. The proof is complete.

Remark 3.2. A question, raised in [13, p. 287], remains open: if $R \in \mathscr{C}$, must the integral closure of $R$ also be in $\mathscr{C}$ ? One might try attacking this question by considering whether the total 2-root closure of $R$, in the sense of [2], is in $\mathscr{C}$. In view of Theorem 2.2 , the construction of the total 2-root closure [2, Proposition 1.1], and the stability of $\mathscr{C}$ under direct limit, an affirmative answer would follow if $\operatorname{dim}(R)=2$ (at least, in characteristic $>2$ ) by showing that 
$R[u] \in \mathscr{C}$ for each $u$ in the quotient field of $R$ such that $u^{2} \in R$. It seems to us that this latter assertion is implausible in general since, in the context of Example 3.1 with characteristic $>2$, one can show that $C=A\left[v_{1}, v_{2}\right]$, for suitable elements $v_{1}, v_{2}$ such that each $v_{i}^{2} \in A$. It follows, under these conditions, that (the non-going-down domain) $B$ is contained in the total 2-root closure of (the divided domain) $A$.

The above points out an important fact, namely, that if $R$ is a domain, $u_{i}$ elements of the quotient field of $R$ such that $u_{i}^{n} \in R$ for some integer $n>1$, and $S$ an overring of $R$ such that $S \subset R\left[\left\{u_{i}\right\}\right]$, then $R \subset S$ need not be a root extension. (As in [1], an extension of domains $R \subset S$ is a root extension if, for each $s \in S$, there exists an integer $n>1$ such that $s^{n} \in R$.) Indeed, if $R \subset S$ is a root extension, then $\operatorname{Spec}(S) \rightarrow \operatorname{Spec}(R)$ is a homeomorphism (cf. [1, Theorem 2.1]); then, since $S$ is integral over $R$, [8, Lemma 2.3] assures that $R \in \mathscr{C}$ if an only if $S \in \mathscr{C}$. However, the preceding paragraph (in conjunction with Example 3.1) produced an extension $A=R \subset B=S \subset C=R\left[v_{1}, v_{2}\right]$ with $v_{i}^{2} \in R, R \in \mathscr{C}$, and $S$ not in $\mathscr{C}$. Accordingly, this $\operatorname{Spec}(S) \rightarrow \operatorname{Spec}(R)$ is not a homeomorphism, and $R \subset S$ is not a root extension. The reader may construct different examples of this phenomenon by using rings of algebraic integers.

The above discussion raises the question of determining conditions under which subrings of the total root closure of a going-down domain are themselves going-down domains. By way of motivation/contrast, we observe, via [8, Lemma 2.3], that if $S$ is the seminormalization (resp., weak normalization) of a going-down domain $R$, then $S$ is a going-down domain, since $\operatorname{Spec}(S) \rightarrow \operatorname{Spec}(R)$ is a homeomorphism.

\section{REFERENCES}

1. D. D. Anderson and M. Zafrullah, Almost Bézout domains, J. Algebra (to appear).

2. D. F. Anderson, D. E. Dobbs, and M. Roitman, Root closure in commutative rings, Ann. Sci. Univ. Clermont-Ferrand II Math. 25 (1990), 1-11.

3. G. Angermüller, On the root and integral closure of Noetherian domains of dimension one, J. Algebra 83 (1983), 437-441.

4. G. Angermüller, Root closure, J. Algebra 90 (1984), 189-197.

5. N. Bourbaki, Commutative algebra, Addison-Wesley, Reading, MA, 1972.

6. D. E. Dobbs, On going-down for simple overrings, II, Comm. Algebra 1 (1974), 439-458.

7. __ Ascent and descent of going-down rings for integral extensions, Bull. Austral. Math. Soc. 15 (1976), 253-264.

8. __ Divided rings and going-down, Pacific J. Math. 67 (1976), 353-363.

9. __ Coherence, ascent of going-down, and pseudo-valuation domains, Houston J. Math. 4 (1978), 551-567.

10. D. E. Dobbs and M. Fontana, Locally pseudo-valuation domains, Ann. Mat. Pura Appl. 134 (1983), 147-168.

11. D. E. Dobbs, M. Fontana, and I. J. Papick, Direct limits and going-down, Comment. Math. Univ. St. Paul. 31 (1982), 129-135.

12. D. E. Dobbs and I. J. Papick, On going-down for simple overrings, III, Proc. Amer. Math. Soc. 54 (1976), 35-38.

13. __ Going-down: a survey, Nieuw Arch. Wisk. 26 (1978), 255-291.

14. M. Fontana, Topologically defined classes of commutative rings, Ann. Mat. Pura Appl. 123 (1980), 331-355. 
15. J. R. Hedstrom and E. G. Houston, Pseudo-valuation domains, Pacific J. Math. 75 (1978), 137-147.

16. W. Heinzer and J. Ohm, The finiteness of $I$ when $R[X] / I$ is $R$-flat. II, Proc. Amer. Math. Soc. 35 (1972), 1-8.

17. I. Kaplansky, Commutative rings, rev. ed., Univ. of Chicago Press, Chicago and London, 1974.

Department of Mathematics, University of Tennessee, Knoxville, Tennessee 379961300

Dipartimento di Matematica, Universita di Roma, "La Sapienza," 00185, Roma, Italy 